\title{
ON THE EXISTENCE OF EQUILIBRUIM STATES OF AN ELASTIC BEAM ON A NONLINEAR FOUNDATION
}

\author{
M.B.M. ELGINDI \\ Department of Mathematics \\ University of Wisconsin-Eau Claire \\ Eau Claire, WI 54702 \\ and \\ D.H.Y. YEN \\ Department of Mathematics \\ Michigan State University \\ East Lansing, MI 48824
}

(Received Received September 23, 1991 and in revised form June 6, 1992)

\begin{abstract}
This paper concerns the existence and uniqueness of equilibrium states of a beamcolumn with hinged ends which is acted upon by axial compression and lateral forces and is in contact with a semi-infinite medium acting as a foundation. The problem is formulated as a fourthorder nonlinear boundary value problem in which the source of the nonlinearity comes from the lateral constraint (the foundation). Treating the equation of equilibrium as a nonlinear eigenvalue problem we prove the existence of a pair of eigenvalue/eigenfunction for each arbitrary prescribed energy level. Treating the equilibrium equation as a nonlinear boundary value problem we prove the existence and uniqueness of solution for a certain range of the acting axial compression force.
\end{abstract}

KEY WORDS AND PHRASES. Existence of equilibrium states, beam-column, elastic beam, fourth-order nonlinear boundary value problem, nonlinear eigenvalue problems, variational methods.

1991 AMS SUBJECT CLASSIFICATION CODES. 49G99, 73H05, 73K15.

\section{INTRODUCTION}

We are concerned in this note with the existence of equilibrium states of a beam-column, i.e., elastic beam under axial compressive forces. The source of the nonlinearity in the present study comes from a nonlinear lateral constraint (foundation). We formulate the problem as a fourthorder nonlinear boundary value problem and prove two theorems on the existence of solution of this boundary value problem. The proofs are based upon a paper by Browder [1].

Existence of solutions of fourth-order boundary value problems arising in the study of equilibrium states of elastic beams has been the subject of several recent papers. Among these are the papers by Agarwal and Chow [2] and Gupta [3]. Both of these papers considered boundary value problems different from the one formulated below. Furthermore, the methods of proofs used there are likewise different from ours. 
We consider a uniform beam-column of length $\ell$ with hinged ends which is acted upon by axial compression $p$ and later force $\tilde{f}(z)$ and is in contact with a semi-infinite elastic medium $\widetilde{k} \widetilde{y}+\widetilde{g}(\widetilde{y})$ acting as a (nonlinear) foundation, (Figure 1). "The modulus of the foundation" $\tilde{k}$ is assumed to be a given non-negative constant.

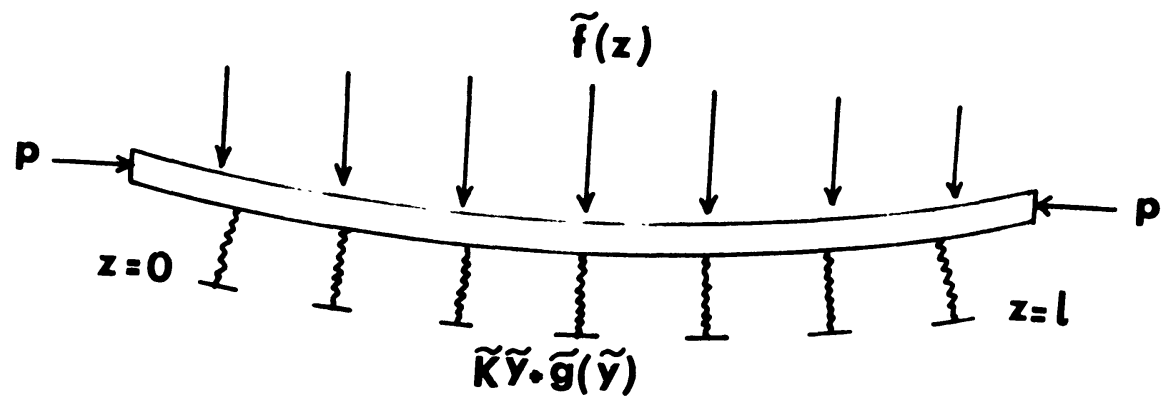

Figure 1

Neglecting geometric and material nonlinearities the governing equation may be taken as

$$
(E I) \frac{d^{4} \widetilde{y}}{d z^{4}}+p \frac{d^{2} \widetilde{y}}{d z^{2}}+\widetilde{k} \widetilde{y}+\widetilde{g}(\widetilde{y})=\tilde{f}(z)
$$

where $\widetilde{y}(z)$ denotes the lateral displacement and $E I$ denotes the bending stiffness.

The boundary conditions for hinged ends are given by

$$
\widetilde{y}(0)=\frac{d^{2} \widetilde{y}}{d z^{2}}(0)=\widetilde{y}(\ell)=\frac{d^{2} \widetilde{y}}{d z^{2}}(\ell)=0 .
$$

Upon using the change of variables

$$
\begin{gathered}
x=\frac{z}{\ell}, \lambda=\frac{p \ell^{2}}{E I}, k=\frac{\widetilde{k} \ell^{4}}{\tilde{E I}}, \\
y(x)=\widetilde{y}(z), g(y)=\frac{\widetilde{g}(\widetilde{y}) \ell^{4}}{E I}, f(x)=\frac{\widetilde{f}(z) \ell^{4}}{E I},
\end{gathered}
$$

the governing equation (1.1) and the boundary conditions (1.2) become

$$
\begin{gathered}
y^{\prime \prime \prime \prime}+\lambda y^{\prime \prime}+k y+g(y)=f(x) \\
y(0)=y^{\prime \prime}(0)=y(1)=y^{\prime \prime}(1)=0
\end{gathered}
$$

where primes in (1.3) and (1.4) signify differentiations with respect to $x$.

In this paper, we are concerned with the question of existence of solution of the fourth-order boundary value problem (1.3), (1.4). We will denote by $H$ the space $W^{2,2}([0,1]) \cap W_{0}^{2,1}([0,1])$. Under the assumptions.

H1: $f \in L^{2}([0,1])$,

H2: there exists $h$ such that $h^{\prime}(u)=g(u), h(u) \in L^{1}([0,1])$ for all $u \in H$ and

(i) For any $C>0$ there exist numbers $\alpha, \beta, \alpha \geq 0$ and $0 \leq \beta<2$, such that

$$
\int_{0}^{1} h(y(x)) d x \geq-\alpha\left\|y^{\prime \prime}\right\|_{L_{2}}^{\beta},
$$

for all $y \in H$ for which $\left\|y^{\prime}\right\|_{L_{2}}^{2}=C$, 
(ii) For any bounded subset $B$ of $H$ there exists $A>0$ such that

$$
\|h(y)-h(z)\|_{L_{1}} \leq A\|y-z\| \text { for all } y, z \in B
$$

it is shown in Section 2 that for any $C>0$ there exists a solution $(\lambda, y)$ of the nonlinear eigenvalue problem (1.3), (1.4) which satisfies the condition $\left\|y^{\prime}\right\|_{L_{2}}^{2}=C$. Under the same assumptions but with $\alpha$ and $\beta$ in H2(i) being independent of $C$ it is shown in Section 3 that for each $\lambda<\pi^{2}$ the nonlinear boundary value problem (1.3), (1.4) has at least one solution and if the nonlinearity $g$ satisfies the additional condition

$$
\int_{0}^{1}\left[g\left(y_{1}\right)-g\left(y_{2}\right)\right]\left(y_{1}-y_{2}\right) d x \geq 0, \text { for all } y_{1}, y_{2} \in H
$$

then the solution is unique. Some discussions are given in Section 4.

2. EXISTENCE OF SOLUTION WITH ARBITRARY ENERGY LEVEL $\left\|y^{\prime}\right\|_{L_{2}}^{2}$.

In this section, we prove that for each $C>0$ there exists $y \in H$ and $\lambda \in \mathbf{R}$ such that

$$
\left\|y^{\prime}\right\|_{L_{2}}^{2}=C
$$

and

$$
\int_{0}^{1} y^{\prime \prime} v^{\prime \prime} d x+\int_{0}^{1}[k y+g(y)] v d x-\int_{0}^{1} f v d s=\lambda \int_{0}^{1} y^{\prime} v^{\prime} d x
$$

for all $v \in H$, i.e., $(\lambda, y)$ is a weak solution for the nonlinear eigenvalue problem (1.3), (1.4) which satisfies (2.1). The regularity theory of [4] then implies that $(\lambda, y)$ is a classical solution of (1.3), (1.4).

For $v, y \in H$ we define the functionals

$$
\begin{aligned}
\Phi(v, y) & =\frac{1}{2} \int_{0}^{1}\left[v^{\prime \prime}\right]^{2} d x+\int_{0}^{1}\left[\frac{k}{2} y^{2}+h(y)\right] d x-\int_{0}^{1} f y d x, \\
E(y) & =\Phi(y, y), \\
g_{1}(y) & =\int_{0}^{1}\left[y^{\prime}\right]^{2} d x .
\end{aligned}
$$

Some properties of the functionals $\Phi, E$, and $g_{1}$ are given in the following Lemmas. These properties enable us to use Theorem 1 of [1] in the proof of the main theorem of this section.

LEMMA 2.1. $\Phi$ is a differentiable and semi-convex on $H \times H$.

PROOF. The differentiability of $\Phi$ as well as the convexity and continuity of $\Phi(\cdot, y)$, for fixed $y$, are clear. Furthermore, if $y_{j} \rightarrow y$ in $H$ for some $\left\{y_{j}\right\}$ then there exist subsequence $y_{j k} \rightarrow y$ in $C^{1}([0,1])$ and hence for fixed $v$ it follows by H2(ii) and Hölder's inequality that there exists a constant $D$ such that

$$
\left|\Phi\left(v, y_{j k}\right)-\Phi(v, y)\right| \leq D\left\|y_{j k}-y\right\|+\|f\|_{L_{2}}\left\|y_{j k}-y\right\|_{L_{2}}
$$

which tends to zero as $k \rightarrow \infty$. This proves that $\Phi\left(v, y_{j}\right) \rightarrow \Phi(v, y)$ for each fixed $v$ and completes the proof for the semi-convexity of $\Phi$.

LEMMA 2.2. $E$ is differentiable on $H$ and $E(y) \rightarrow \infty$ as $\|y\| \rightarrow \infty$ on the set

$$
S=\left\{y \in H:\left\|y^{\prime}\right\|_{L_{2}}^{2}=C\right\} .
$$

PROOF. The differentiability of $E$ follows from that of $\Phi$ since

$$
E^{\prime}(y)=\Phi_{1}^{\prime}(y, y)+\Phi_{2}^{\prime}(y, y)
$$


Also from the definition of $E$ is follows (by the properties of $y \in H$ and H2(i)) that

$$
E(y) \geq \frac{1}{2}\left\|y^{\prime \prime}\right\|_{L_{2}}^{2}-\alpha\left\|y^{\prime \prime}\right\|_{L_{2}}^{\beta}-C_{1}\|f\|\left\|y^{\prime \prime}\right\|_{L_{2}}+\frac{k}{2} \int_{0}^{1} y^{2} d x,
$$

for some $\alpha \geq 0,0 \leq \beta<2$ and some constant $C_{1}>0$. Now since $k \geq 0$ we have

$$
E(y) \geq \frac{1}{2}\left\|y^{\prime \prime}\right\|_{L_{2}}^{2}-\alpha\left\|y^{\prime \prime}\right\|_{L_{2}}^{\beta}-C_{1}\|f\|\left\|y^{\prime \prime}\right\|_{L_{2}} .
$$

Thus $E(y) \rightarrow \infty$ as $\|y\| \rightarrow \infty$ on $S$ (since on $S$ we have $\|y\| \rightarrow \infty$ if and only if $\left\|y^{\prime \prime}\right\|_{L_{2}} \rightarrow \infty$ and $\beta<2$ ).

LEMMA 2.3. $g_{1}$ is weakly continuous on $H$.

PROOF. Suppose that $y_{j} \rightarrow y$ in $H$. Then there exists a subsequence $\left\{y_{j k}\right\}$ such that $y_{j k} \rightarrow y$ in $C^{1}([0,1])$ and hence $g_{1}\left(y_{j k}\right) \rightarrow g_{1}(y)$. Thus $g_{1}\left(y_{j}\right) \rightarrow g_{1}(y)$.

THEOREM 2.4. For each $C>0$ there exist some $y \in H$ and $\lambda \in R$ satisfying (2.1) and (2.2).

PROOF. It follows from Lemma 2.3 that the set

$$
S=\left\{y \in H:\left\|y^{\prime}\right\|_{L_{2}}^{2}=C\right\}
$$

is weakly closed. From Lemmas 2.1 and 2.2 we see that $\Phi$ and $E$ satisfy the conditions of Theorem 1 of [1]. It follows that $E$ attains its minimum at some $y \in S$. From Lusternik's Theorem it follows that there exist $\lambda \in \mathbf{R}$ such that $\lambda$ and $y$ satisfy

$$
E^{\prime}(y)=\lambda g_{1}^{\prime}(y)
$$

which implies that for these $\lambda$ and $y$ equation (2.2) is satisfied for all $v \in H$. Since $y \in S$ it follows that $y$ satisfies (2.1).

3. EXISTENCE OF SOLUTION FOR FIXED $\lambda$ :

In this section, we consider the existence of solution of the fourth order nonlinear boundary value problem (1.3), (1.4) with $\lambda$ being fixed. Under the same assumptions $\mathrm{H} 1$ and H2 but with $\alpha$ and $\beta$ in H2(i) being independent of $C$ it is proved that for each $\lambda<\pi^{2}, k \geq 0$ there exists $y \in H$ such that

$$
\int_{0}^{1} y^{\prime \prime} v^{\prime \prime} d x+\int_{0}^{1}[k y+g(y)] v d x-\int_{0}^{1} f v d x-\lambda \int_{0}^{1} y^{\prime} v^{\prime} d x=0
$$

for all $v \in H$, i.e., $y$ is a weak solution of (1.3), (1.4). The proof is again based upon the variational methods of [1].

We define the functionals $\Phi$ and $E$ on $H \times H$ and $H$ respectively by

$$
\begin{aligned}
& \Phi(v, y)=\frac{1}{2} \int_{0}^{1}\left[v^{\prime \prime}\right]^{2} d x-\frac{\lambda}{2} \int_{0}^{1}\left[y^{\prime}\right]^{2} d x+\int_{0}^{1}\left[\frac{k}{2} y^{2}+h(y)\right] d x-\int_{0}^{1} f y d x, \\
& E(y)=\Phi(y, y),
\end{aligned}
$$

and examine some of their properties in the following lemma.

LEMMA 3.1. The functionals $\Phi$ and $E$ are differentiable, $\Phi$ is semi-convex and $E$ is coercive, i.e., $E(y) \rightarrow \infty$ as $\|y\| \rightarrow \infty$, provided that $k \geq 0$ and $\lambda<\pi^{2}$.

PROOF. Since $\Phi$ is clearly differentiable, continuous and convex with respect to $v$ for each fixed $y$ and since the imbedding of $H$ onto $C^{1}([0,1])$ is compact it follows by H2(ii) and Hölder's inequality (as in Lemma 2.1) that it is semi-convex. The differentiability of $E$ is also clear. Now using $k \geq 0, \mathrm{H} 2(\mathrm{i})$ and the inequality

it follows from the definition of $E$ that

$$
\left\|y^{\prime}\right\|_{L_{2}}^{2} \leq \frac{1}{\pi^{2}}\left\|y^{\prime \prime}\right\|_{L_{2}}^{2}
$$




$$
E(y) \geq \frac{1}{2}\left(1-\frac{\lambda}{\pi^{2}}\right)\left\|y^{\prime \prime}\right\|_{L_{2}}^{2}-\alpha\left\|y^{\prime \prime}\right\|_{L_{2}}^{\beta}-\|f\|_{L_{2}}\|y\|_{L_{2}}
$$

which implies (since $\beta<2$ and $\lambda<\pi^{2}$ ) that $E$ is coercive.

THEOREM 3.2. For each $k \geq 0$ and $\lambda<\pi^{2}$ the boundary value problem has a solution. Furthermore, if the nonlinearity $g$ satisfies the additional condition

$$
\int_{0}^{1}\left[g\left(y_{1}\right)-g\left(y_{2}\right)\right]\left(y_{1}-y_{2}\right) d x \geq 0
$$

for all $y_{1}, y_{2} \in H$ with $y_{1} \neq y_{2}$, then the solution is unique.

PROOF. Lemma 3.1 enables us to apply Theorem 1 of [1] which implies the existence of a solution $y$ of (3.1). The smoothness of $y$ follows from the regularity theory of [4]. Finally, if (3.2) holds it can be easily checked that (3.1) can have at most one solution for each $k \geq 0$ and $\lambda<\pi^{2}$.

4. DISCUSSIONS.

We remark that the parameters $\lambda$ and $C$ of Theorem 2.4 represent the dimensionless axial compression acting on the beam-column and a part of the total bending energy for the beamcolumn respectively.

Many important nonlinearities $g(y)$ encountered in practice satisfy the condition $\mathrm{H} 2$ which was used in the proofs of Theorems 2.4 and 3.2. Among such $g(y)$ are the polynomials. To see this we note that if $g(y)=y^{r}, r \geq 2$, where $r$ is odd then $h(y)=\frac{1}{r+1} y^{r+1} \geq 0$ and H2(i) holds trivially by taking $\alpha=\beta=0$. If $r$ is even, then $r+1$ is odd and we have

However,

$$
\int_{0}^{1} y^{r+1} d x=\int_{0}^{1} y^{r} y d x=\int_{0}^{1} y^{2 k} y d x
$$

$$
\begin{aligned}
y^{2}(x) & =2 \int_{0}^{x} y y^{\prime} d s \leq 2\|y\|_{L_{2}}\left\|y^{\prime}\right\|_{L_{2}} \\
& \leq 2 C^{1 / 2}\|y\|_{L_{2}} \\
& \leq 2 \frac{C^{1 / 2}}{\pi}\left\|y^{\prime}\right\|_{L_{2}}=\frac{2 C}{\pi}
\end{aligned}
$$

where $C=\left\|y^{\prime}\right\|_{L_{2}}^{2}$. Thus

$$
\left|\int_{0}^{1} y^{r+1} d x\right| \leq C_{1}
$$

for some constant $C_{1}$ and hence $g(y)=y^{r}$ satisfies H2(i) for all $r$ with $\beta=0$. Condition H2(ii) is clearly satisfied for $g(y)=y^{r}$ with $r$ odd or even. Thus condition H2 is satisfied when $g(y)$ is of polynomial function of $y$. We also note that $\alpha$ and $\beta$ are independent of the constant $C$ when $g(y)$ is an odd power of $y$ and that condition (3.2) of Theorem 3.2 is satisfied in this case. Thus if $g(y)$ is an odd power of $y$ and $\lambda<\pi^{2}$, the nonlinear boundary value problem (1.3), (1.4) has a unique solution. Finally, we remark that the number $\pi^{2}$ is the smallest eigenvalue of the linear boundary value problem

$$
\begin{gathered}
y^{\prime \prime \prime \prime}+\lambda y^{\prime \prime}+k y=0, \\
y(0)=y^{\prime \prime}(0)=y(1)=y^{\prime \prime}(1)=0
\end{gathered}
$$

when $k=0$ and hence for $\lambda$ beyond $\pi^{2}$ bifurcation of solution may take place. A bifurcation analysis of the solution set in neighborhood of the first eigenvalue of (4.1), (4.2) will be given in a 
when $k=0$ and hence for $\lambda$ beyond $\pi^{2}$ bifurcation of solution may take place. A bifurcation analysis of the solution set in neighborhood of the first eigenvalue of $(4.1),(4.2)$ will be given in a forthcoming paper.

\section{REFERENCES}

1. BROWDER, F. "Variational methods for nonlinear elliptic eigenvalue problems," Bull. Amer. Math. Soc. 71 (1965), 176-183.

2. RAVI, P. and CHOW, Y.M. "Iterative methods for fourth-order boundary value problems," J. Comp. Appl. Math. 10 (1984), 203-207.

3. GUPTA, C.P. "Solvability of fourth-order boundary value problem with periodic boundary conditions," Internat. J. Math. \& Math. Sci. Vol. 11, No. 2 (1988), 275-284.

4. MORREY, C.B. "On the analyticity of analytic nonlinear elliptic system of partial differential equations," I, II, Am. J. Math. 8 (1958), 198-237. 


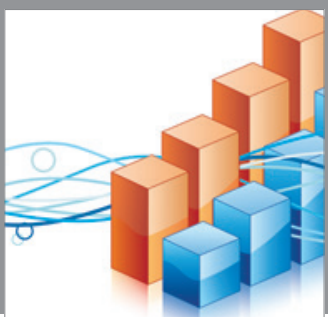

Advances in

Operations Research

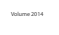

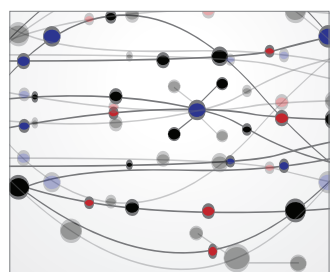

\section{The Scientific} World Journal
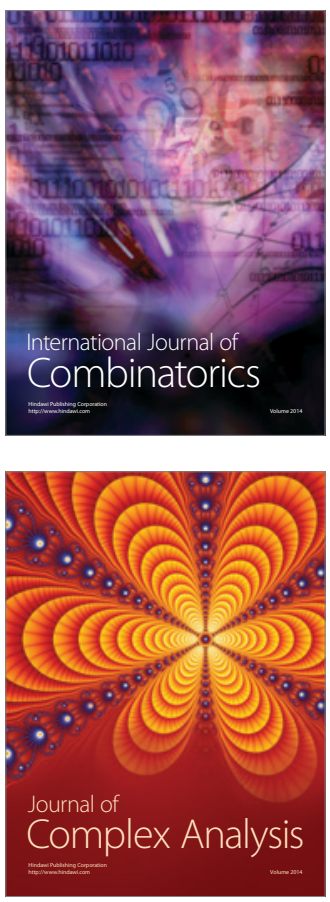

International Journal of

Mathematics and

Mathematical

Sciences
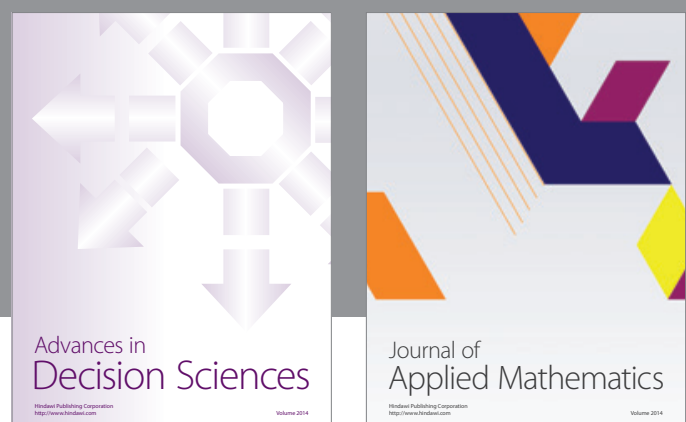

Journal of

Applied Mathematics
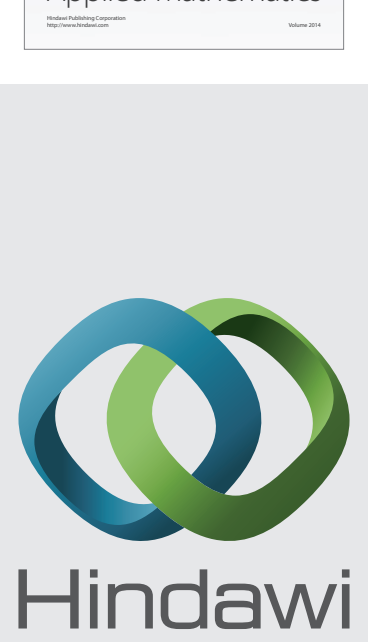

Submit your manuscripts at http://www.hindawi.com
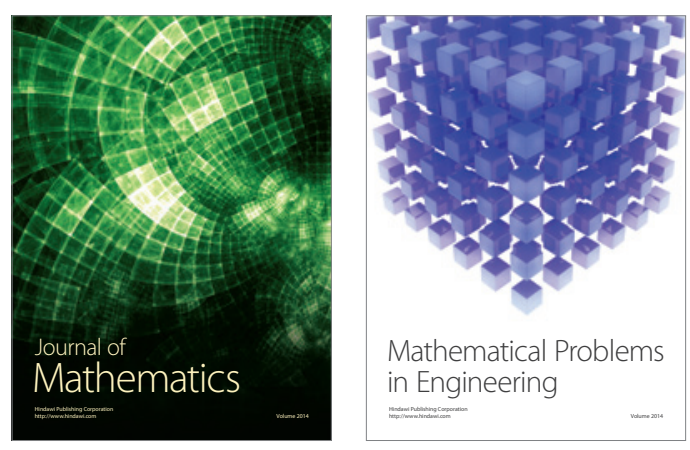

Mathematical Problems in Engineering
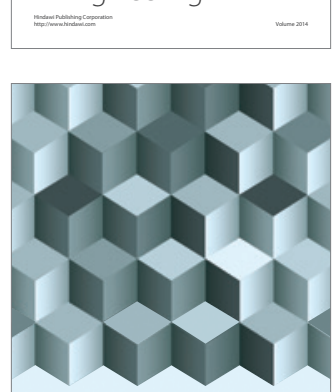

Journal of

Function Spaces
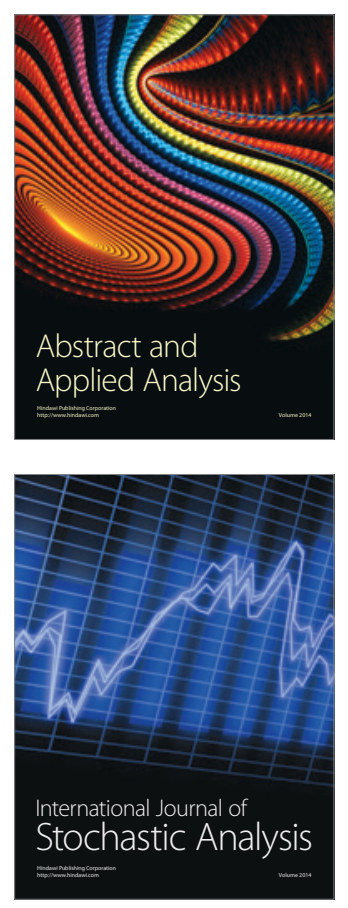

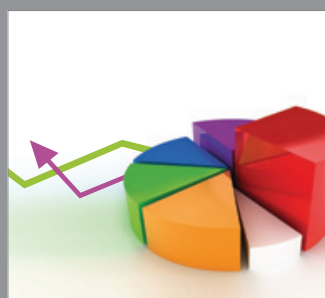

ournal of

Probability and Statistics

Promensencen
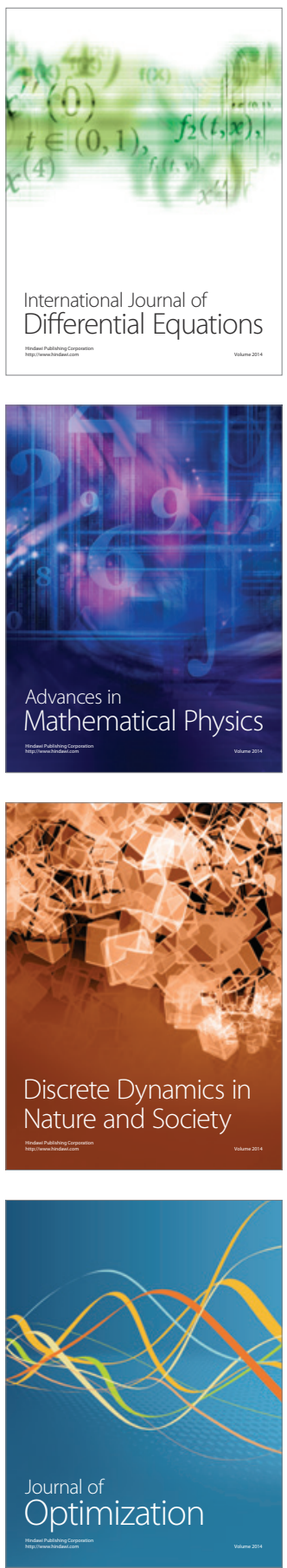